\title{
Diffuse small bowel thickening in Aids patient - a case report
}

\author{
Rohit Singla', Samriti Hari², Surendra K Sharma ${ }^{1 *}$
}

\begin{abstract}
Background: Diarrhea is common in HIV/AIDS patients, caused by both classic enteric pathogens and different opportunistic agents. Infection with these different pathogens may lead to similar radiological findings, thus causing diagnostic confusion.

Case presentation: A 30-yr-old female with AIDS presented with chronic diarrhea of 4 months duration. She had diffuse small bowel thickening present on $\mathrm{CT}$ scan of her abdomen, with stool examination showing no parasites. She was erroneously diagnosed as abdominal tuberculosis and given antituberculosis drugs with which she showed no improvement. Repeat stool examination later at a specialized laboratory revealed Cryptosporidium parvum infection.

The patient was given an extended course of nitazoxanide treatment, as her stool examination was positive for Cryptosporidium parvum even after 2 weeks of drug consumption. Parasite clearance was documented after 10 weeks of treatment. Interestingly, the bowel thickening reversed with parasitological clearance.
\end{abstract}

Conclusions: Cryptosporidium parvum may lead to small bowel thickening in AIDS patients. This small bowel thickening may reverse following parasitological clearance.

\section{Background}

Diarrhea is a common manifestation of AIDS [1,2] (acquired immunodeficiency syndrome), causing significant morbidity and mortality. Diarrhea in AIDS patients is caused by both classic enteric pathogens and different opportunistic agents. Infection with these different pathogens may lead to similar radiological findings, thus causing diagnostic confusion.

Cryptosporidium parvum is a common cause of diarrhea in AIDS patients [3]. The fact that it may cause diffuse small bowel thickening has been reported by Redvanly et al [4], but is not widely recognized. Limited treatment options are available against this parasite. Nitazoxanide is the only drug approved for treatment of this disease. However, there is not much data supporting its efficacy in immunocompromised individuals. Also, there are no clear guidelines stating the exact duration of treatment with this drug.

\footnotetext{
* Correspondence: surensk@gmail.com

'Department of Medicine, All India Institute of Medical Sciences, New Delhi, India

Full list of author information is available at the end of the article
}

\section{Case presentation}

A 30-yr-old female, diagnosed case of AIDS, was admitted for evaluation of weight loss and intractable diarrhea of 4 months duration. The patient complained of chronic, painless, nonbloody, profuse watery diarrhea with a weight loss of $10 \mathrm{~kg}$ (decrease to $30 \mathrm{~kg}$ from $40 \mathrm{~kg}$ prior to illness). She had no complaints of fever, cough or expectoration.

Previously, during evaluation of her complaints in the outpatient department, she was discovered to be HIV (human immunodeficiency virus) infected with a CD4 count of 38 cells $/ \mu$ l. She also had anemia with hemoglobin value of $8 \mathrm{~g} / \mathrm{dl}$, normal kidney function, normal AST/ALT (aspartate aminotransferase/alanine aminotransferase) and elevated serum alkaline phosphatase. Stool examination showed no red blood cells or leukocytes, and no ova or cysts were observed. A contrast enhanced CT scan of the abdomen (figure 1) showed diffuse small bowel wall thickening, a fatty liver and no significant lymphadenopathy or ascites. Her chest radiograph was normal. Based on these details, a diagnosis of abdominal tuberculosis was made 2 months back, and she was started on standard 4-drug regimen for 


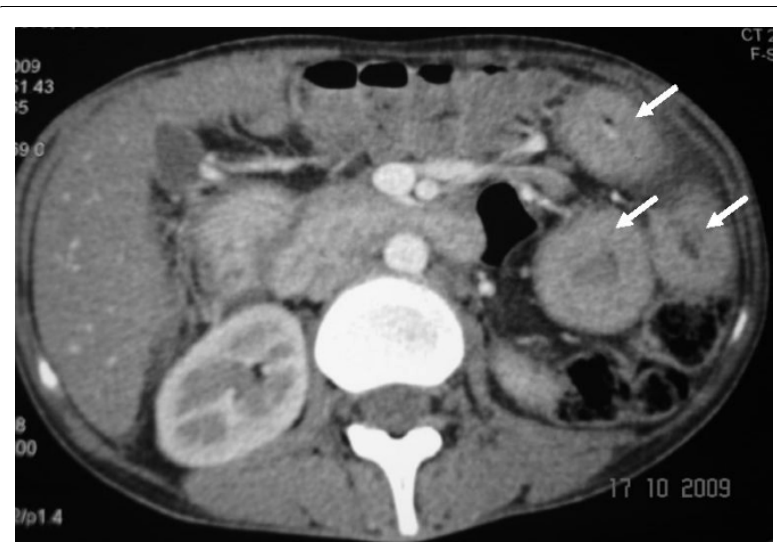

Figure 1 Axial contrast enhanced CT image shows diffuse, concentric wall thickening of multiple small bowel loops (arrows)

treatment of tuberculosis. Besides this, she was also given co-trimoxazole and once a week azithromycin prophylaxis for Pneumocystis jiroveci and mycobacterium avium-intracellulare infection respectively. Two weeks later she was started on non-zidovudine based HAART (highly active antiretroviral therapy), after she had tolerated antituberculosis drugs. However diarrhea failed to improve, and she continued to lose weight.

During this admission her stool sample was sent to a specialized microbiology laboratory, where acid fast oocysts of Cryptosporidium parvum were observed. Her antituberculosis drugs were stopped and she was started on treatment with tablet Nitazoxanide $500 \mathrm{mg}$ twice a day. Her peripheral smear showed a megaloblatic anemia (mean corpuscular volume - $120 \mathrm{fl}$ ), for which folate and vitamin $B_{12}$ were supplemented. Her kidney functions, AST/ALT were again normal, but she had raised serum alkaline phosphatase and a low serum albumin of $2.8 \mathrm{~g} / \mathrm{dl}$. In hospital she was also given supportive care with oral rehydration solution, antidiarrhoeals, and a nutritious diet low in lactose. After 2 weeks of nitazoxanide treatment the patient's stool remained positive for cryptosporidium. Her hemoglobin level had improved with folate and vitamin $B_{12}$ supplementation. At this moment it was decided to discharge her on extended nitazoxanide treatment while continuing HAART, antibiotic prophylaxis and folate and vitamin $B_{12}$, with monthly follow up.

Patient was again observed 2 months after discharge. Her diarrhea had settled for last 1 week. Her stool this time was negative for Cryptosporidium parvum, which was confirmed twice. Repeat CT scan of the abdomen (figure 2) showed resolution of the small bowel thickening, persistent fatty liver, no lymphadenopathy or ascites. A repeat $C D 4$ count was done which was 36 cells/ $\mu$ l only.

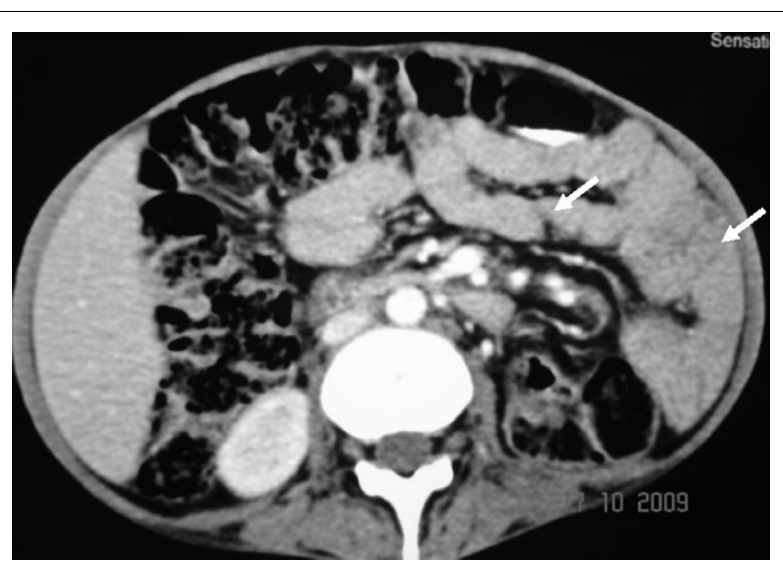

Figure 2 Axial contrast enhanced CT image shows normal small bowel loops after 10 weeks of antiparasitic therapy (arrows).

Following parasitological clearance, the patient showed improvement in appetite and gained $5 \mathrm{~kg}$ weight over next one month. HAART and vitamin supplementation was continued. In view of lack of definite guidelines on secondary prophylaxis, the authors decided to continue nitazoxanide until the patient would achieve a CD4 count of 100 cells/ $\mu$ l.

\section{Discussion}

The number of HIV infected individuals is continuing to increase worldwide. World Health Organization estimated that 33 million individuals worldwide were HIV infected at the end of year 2007 [5]. Diarrhea is a common manifestation of this disease, causing significant morbidity and mortality $[2,3]$. Superimposed infection by both classic pathogens and different opportunistic agents results due to defective immunity, leading to diarrhea.

Cryptosporidium is a protozoan parasite which causes a self-limited diarrhoeal illness in immunocompetent individuals and chronic, intractable diarrhea in AIDS patients; especially those with a CD4 count $<100 /$ ul. Typically, diarrhea is profuse, watery, nonbloody, leading to fluid and electrolyte depletion. Stool examination shows no red blood cells or leukocytes. In a recently done study at Pune in India [6], 16 (12\%) of 137 consecutive HIV infected patients with diarrhea had Cryptosporidium parvum infection.

Cryptosporidial infection has a predilection for the proximal small bowel, resulting in nonspecific thickening of the duodenum, jejenum and proximal ileum [4]. Multiple loops of fluid-filled and thickened small bowel loop can be identified on CT. Lymphadenopathy is not a feature of the disease [4]. Infection with Isospora belli can result in a gastrointestinal disease that is clinically and radiologically indistinguishable from cryptosporidiosis 
[7]. Small bowel thickening in AIDS patients may also be seen due to small bowel lymphoma [8] or infection with mycobacterium avium-intracellulare $[9,10]$, both of which are associated with lymphadenopathy. Mycobacterium tuberculosis infection results in necrotic lymph nodes on CT in $90 \%$ of patients, with focal hepatic and splenic lesions, ascites and distal ileal thickening [9]. Hence, the finding of diffuse small bowel wall thickening with no lymphadenopathy and no focal lesions in liver/ spleen in our case argued against a diagnosis of tuberculosis.

Our case also shows that the small bowel thickening seen on CT abdomen resolved with parasitological clearance. Such a result is expected, but not previously reported in English literature.

Limited treatment options are available which have shown success in eradicating this parasite in immunocompromised patients. In a double-blind randomised controlled trial in Mexico [11], nitazoxanide 1-2 g daily for 2 weeks resulted in parasitologic cure in $65 \%$ of treated patients. Another trial done at United States [12] supported the use of nitazoxanide in AIDS patients with diarrhoea. However, a recently conducted Cochrane review [13] which included 7 trials involving 169 patients showed no reduction in the duration or frequency of diarrhoea by nitazoxanide compared with placebo. Also, there was a lot of heterogeneity in drug dosage and duration of treatment. The CDC guidelines [14]presently recommend nitazoxanide along with antiretroviral treatment for AIDS patients with cryptosporidiosis. These antiparasitic drugs, however, should never be used as a substitute for HAART in the treatment of AIDS-associated cryptosporidiosis.

Our case shows that extended treatment with nitazoxanide may lead to parasitological clearance, even in face of low CD4 count. In our case, the lack of rise in CD4 count, despite consuming 4 months of HAART can be explained by various factors. It could be ascribed to malabsorption of drugs due to chronic diarrhea. Additionally, non-adherence may also explain poor response to HAART in addition to malabsorption. Lack of rise in CD4 count supports the notion that parasitological clearance was likely because of nitazoxanide, and not secondary to immune reconstitution. However, change in CD4 count is an indirect measure of efficacy of HAART. This observation in the absence of demonstration of concomitant rise in viral load does not definitively prove that HAART was not efficacious.

Paromomycin and azithromycin have also been tried, with little data supporting their efficacy. Best treatment option remains immune reconstitution with HAART. This results in excellent clinical responses as assessed by stool frequency, weight gain, and clearance of oocysts from the stool. However, rapid relapse after discontinuation of antiretroviral therapy suggests that cryptosporidial infection is suppressed rather than cured $[15,16]$. Supportive care with antidiarrhoeals, correction of fluid and electrolyte disturbance, and a nutritious diet low in lactose should also be provided to these patients.

\section{Conclusions}

Cryptosporidium parvum is a frequent cause of diarrhea in AIDS patients, especially those with a CD4 count $<100 /$ ul. It may lead to small bowel thickening, which should not be ascribed to other etiologies in an appropriate clinical setting. This small bowel thickening may reverse following parasitological clearance. Limited treatment options are available against this pathogen. Extended treatment with nitazoxanide along with HAART may help in achieving parasitological clearance.

\section{Consent}

Written informed consent was obtained from the patient.

\section{Abbreviations}

AIDS: acquired immunodeficiency syndrome; ALT: alanine aminotransferase; AST: aspartate aminotransferase; HIV: human immunodeficiency virus;

\section{Acknowledgements}

The authors will like to thank the patient. We are also grateful to Dr. Manish Soneja and Mrs Rekha Sharma who were involved in caring for the patient and in writing of this case report.

\section{Author details}

${ }^{1}$ Department of Medicine, All India Institute of Medical Sciences, New Delhi, India. ${ }^{2}$ Department of Radio Diagnosis, All India Institute of Medical Sciences, New Delhi, India, Study conducted at All India Institute of Medical Sciences, New Delhi-110029, India.

\section{Authors' contributions}

RS was involved in patient care, and was a major contributor in writing the manuscript. SH was the radiologist who interpreted the CT films. SKS was involved in writing the manuscript and patient care. All authors have read and approved the final manuscript

\section{Competing interests}

The authors declare that they have no competing interests.

Received: 30 December 2009 Accepted: 28 October 2010 Published: 28 October 2010

\section{References}

1. Janoff EN, Smith PD: Prospectives on gastrointestinal infections in AIDS. Gastroenterol Clin North Am 1988, 17:451-63.

2. Framm SR, Soave R: Agents of diarrhea. Med Clin North Am 1997, 81:427-47.

3. Wiwanitkit $\mathrm{V}$ : Intestinal parasitic infections in Thai HIVinfected patients with different immunity status. BMC Gastroenterol 2001, 1:3-5.

4. Redvanly RD, Silverstein JE: Intra-abdominal manifestations of AIDS. Radiol Clin North Am 1997, 35:1083-125.

5. UNAIDS: 2008 report on the global AIDS epidemic. Geneva; UNAIDS; 2008.

6. Kulkarni SV, Kairon R, Sane SS, Padmawar PS, Kale VA, Thakar MR, Mehendale SM, Risbud AR: Opportunistic parasitic infections in HIV/AIDS patients presenting with diarrhoea by the level of immunesuppression. Indian J Med Res 2009, 130:63-66. 
7. Goodgame RW: Understanding intestinal spore forming protozoa: cryptosporidia, microsporidia,isospora and cyclospora. Ann Intern Med 1996, 124:429-41.

8. Radin DR, Esplin JA, Levine AM, Ralls PW: AIDS related non-Hodgkin's lymphoma: abdominal CT findings in 112 patients. AJR 1993, 160:1122-39.

9. Radin DR: Intraabdominal Mycobacterium tuberculosis vs Mycobacterium avium-intracellulare infections in patients with AIDS: distinction based on CT findings. AJR 1991, 156:487-91.

10. Nyberg DA, Federle MP, Jeffrey RB, Bottles K, Wofsy CB: Abdominal CT findings of disseminated Mycobacterium avium-intracellulare in AIDS. AJR 1985, 145:297-9.

11. Rossignol JF, Hidalgo H, Feregrino M, Higuera F, Gomez WH, Romero JL, Padierna J, Geyne A, Ayers MS: A double-blind placebo-controlled study of nitazoxanide in the treatment of cryptosporidial diarrhoea in AIDS patients in Mexico. Trans R SocTrop Med Hyg 1998, 92:663-66.

12. Rossignol JF: Nitazoxanide in the treatment of acquired immune deficiency syndrome-related cryptosporidiosis: results of the United States compassionate use program in 365 patients. Aliment Pharmacol Ther 2006, 24:887-94

13. Abubakar II, Aliyu SH, Arumugam C, Hunter P, Usman N: Prevention and treatment of cryptosporidiosis in immunocompromised patients. Cochrane Database of Systematic Reviews 2007, 1: CD004932.

14. Treating Opportunistic Infections Among HIV-Infected Adults and Adolescents. [http://www.cdc.gov/mmwr/preview/mmwrhtml/rr5315a1. htm].

15. Carr A, Marriott D, Field A, Vasak E, Cooper DA: Treatment of HIV-1associated microsporidiosis and cryptosporidiosis with combination antiretroviral therapy. Lancet 1998, 351:256-61.

16. Maggi P, Larocca AM, Quarto M, Serio G, Brandonisio O, Angarano G, Pastore G: Effect of antiretroviral therapy on cryptosporidiosis and microsporidiosis in patients infected with human immunodeficiency virus type 1. Eur J Clin Microbiol Infect Dis 2000, 19:213-17.

\section{Pre-publication history}

The pre-publication history for this paper can be accessed here: http://www.biomedcentral.com/1471-2334/10/310/prepub

doi:10.1186/1471-2334-10-310

Cite this article as: Singla et al.: Diffuse small bowel thickening in Aids patient - a case report. BMC Infectious Diseases 2010 10:310.

\section{Submit your next manuscript to BioMed Central and take full advantage of:}

- Convenient online submission

- Thorough peer review

- No space constraints or color figure charges

- Immediate publication on acceptance

- Inclusion in PubMed, CAS, Scopus and Google Scholar

- Research which is freely available for redistribution

Submit your manuscript at www.biomedcentral.com/submit
Biomed Central 